\title{
Increased serum levels of interleukin-17 in patients with alopecia areata and non-segmental vitiligo
}

\author{
Katarzyna A. Tomaszewska¹, Magdalena Kozłowska², Andrzej Kaszuba², Aleksandra Lesiak², Joanna Narbutt², \\ Anna M. Zalewska-Janowska ${ }^{1}$
}

${ }^{1}$ Psychodermatology Department, Pulmonology, Rheumatology and Clinical Immunology Chair, Medical University of Lodz, Lodz, Poland 2Department of Dermatology, Pediatric Dermatology and Oncology, Medical University of Lodz, Lodz, Poland

Adv Dermatol Allergol 2022; XXXIX (1): 195-199

DOI: https://doi.org/10.5114/ada.2022.113612

\begin{abstract}
Introduction: Alopecia areata $(\mathrm{AA})$ and vitiligo are both skin diseases of autoimmune origin. AA is characterized by patchy hair loss primarily on the scalp but may involve other areas as well, while vitiligo is caused by the destruction of melanocytes and results in the appearance of white patches on any part of the body. Many facts indicate similar pathogenesis of these diseases. Both dermatoses are associated with frequent coexistence of other autoimmune diseases and share common genetic risk factors. Recent data support the theory of the involvement of IL-17 in the pathogenesis of both $A A$ and vitiligo.

Aim: To evaluate and compare the serum levels of interleukin (IL)-17 in patients with AA and non-segmental vitiligo (NSV). To assess whether the pattern of serum cytokine concentration can be associated with clinical details and activity of the disease.

Material and methods: A cross-sectional study was conducted on 33 patients with AA, 30 patients with NSV, and 30 healthy controls. Serum levels of IL-17 were determined quantitatively by ELISA method.

Results: Our analysis identified a systemic inflammatory signature associated with AA and NSV, characterized by elevated levels of IL-17. The levels of IL-17 did not differ significantly between AA patients and NSV patients.

Conclusions: Our results show that AA and vitiligo may share common etiopathogenetic pathways, which suggests the potential of developing targeted therapies for both AA and vitiligo treatment. Imbalance of T cell subpopulations and complex systemic cytokine profiles may contribute to the pathogenesis of AA and vitiligo.
\end{abstract}

Key words: alopecia areata, vitiligo, cytokines, interleukin, interleukin-17.

\section{Introduction}

Alopecia areata (AA) is a non-scarring hair loss with an unpredictable course and a wide spectrum of manifestations [1]. The pathogenesis of AA is considered an organ-specific autoimmune process with a genetic background. The hair follicle is an immune-privileged site, with low levels of major histocompatibility complex (MHC) expression. It is assumed that AA results from a breakdown in immune privilege with the subsequent assault on the follicle on the level of the bulb by CD8+ $\mathrm{T}$ lymphocytes. The peribulbar lymphocytic infiltrate induces hair follicle keratinocytes to undergo apoptosis that results in inhibition of cell division within the hair matrix and hair shaft production [2].

Vitiligo is an acquired disorder of the skin and mucous membranes that is characterized by well-circum- scribed, depigmented macules, and patches that occur secondary to selective destruction of melanocytes. There are several pathophysiologic theories; the most prominent are autoimmune, neurohumoral, and autocytotoxic. The convergence theory states that stress, accumulation of toxic compounds, infection, autoimmunity, mutations, altered cellular environment, and impaired melanocyte migration can all contribute to pathogenesis [3].

$A A$ and vitiligo are both autoimmune diseases, and striking similarities in pathogenesis have been identified at the level of both the innate and adaptive immune system. In recent years, different studies have tried to associate the interleukin-17 (IL-17) pathway to an increasing number of inflammatory diseases, such as psoriasis, rheumatoid arthritis, multiple sclerosis, inflammatory bowel disease, systemic sclerosis, primary Sjogren's syndrome [4]. However, the role of IL-17 in AA, and vitiligo is still unclear.

Address for correspondence: Katarzyna A. Tomaszewska PhD, Psychodermatology Department, Pulmonology, Rheumatology and Clinical Immunology Chair, Medical University of Lodz, Lodz, Poland, phone: +48 508 126 620, e-mail: katarzyna.tomaszewska@umed.lodz.pl Received: 21.11.2020, accepted: 24.12.2020. 


\section{Aim}

In a previous study [5], it was reported that interferon- $\gamma$ (IFN- $\gamma$ ), IL-1 $\beta$, and IL- 6 serum levels were significantly elevated in AA patients and non-segmental vitiligo (NSV) patients compared to healthy controls. The IL-6 level was also significantly higher in NSV compared to AA patients. In this study, we aimed to determine and compare the serum levels of IL-17 in AA and NSV patients. For this, we concurrently measured the serum levels of IL-17 in patients with AA and NSV. Whether the pattern of serum cytokine concentration could be associated with clinical details and disease activity in patients was also investigated. The results of this study may help to improve AA and vitiligo therapeutic strategies by targeting cytokines involved in disease pathogenesis.

\section{Material and methods}

\section{Material}

The study included 33 patients with AA and 30 patients with NSV who were hospitalized in the Department of Dermatology, Paediatric Dermatology and Oncology from October 2016 to June 2019. The control group consisted of 30 healthy individuals. The diagnosis of AA and NSV was based on a detailed medical history, clinical and dermoscopic/trichoscopic examination. Exclusion criteria were (a) systemic immunosuppressant therapy within the past 4 weeks; (b) history of topical treatment within the past 2 weeks; and (c) clinically active infection. The control group was selected based on demographic similarities to the research group.

The group of patients with AA included 21 (63.6\%) females and 12 (36.4\%) males and the group of patients with vitiligo included 18 (60\%) females and 12 (40\%) males. The control group contained 23 (76.7\%) females and 7 (23.3\%) males. The mean ages of AA patients, NSV patients, and healthy controls were $18.64 \pm 8.56$ years, $28.55 \pm 19.23$ years and $19.95 \pm 13.08$ years, respectively. There were no statistical differences in sex ( $\chi^{2}$ test) and age ( $t$ test) between the patients and the normal controls.
To assess the clinical extent of AA and NSV, we calculated Severity of Alopecia Tool (SALT) [6] and Vitiligo Area Severity Index (VASI) [7] scores, respectively. Activity of both AA and NSV was assessed using Vitiligo Disease Activity Score (VIDA) [8].

The study was approved by the Bioethics Committee of the Medical University of Lodz (RNN/266/16/KE) and was conducted following the Declaration of Helsinki. All subjects gave informed consent to participate in the study.

\section{Methods}

Peripheral venous blood samples were collected from all patients and healthy controls. Sera were isolated by centrifugation and stored at $-70^{\circ} \mathrm{C}$ before analysis and the concentrations of IL-17 [pg/ml] (Diaclone, Besancon Cedex, France) were determined quantitatively in collected serum samples by the enzyme-linked immunosorbent assay (ELISA) method in both patients and controls. ELISA tests were performed according to the manufacturer's instructions. The detection range of IL-17 was 7.8-500 pg/ $\mathrm{ml}$. The serum cytokine levels were compared between groups. The correlation of serum cytokine levels with sex, extent, activity, and duration of disease, associated autoimmune disorders, atopic dermatitis (AD) and family history was studied.

\section{Statistical analysis}

Statistical analysis was carried out with the Statistica software version 12 (StatSoft, Tulsa, OK, USA). To determine the distribution of quantitative variables, the Shapiro-Wilk test was used. The Mann-Whitney test was used to compare the median serum cytokine levels between the groups. Correlation analysis was determined using the Spearman rank correlation test. Data were considered to be statistically significant at a value of $p<0.05$.

\section{Results}

The demographic and clinical characteristics of study participants are described in Table 1. Of the 33 enrolled

Table 1. Demographic and clinical characteristics of patients with alopecia areata (AA), patients with non-segmental vitiligo (NSV) and healthy controls

\begin{tabular}{lccc}
\hline Parameter & AA patients & NSV patients & Controls \\
\hline Total & 33 & 30 & 30 \\
\hline Female/male: & & & $23 / 7$ \\
\hline$n$ & $21 / 12$ & $18 / 12$ & $76.7 / 23.3$ \\
\hline$\%$ & $63.6 / 36.4$ & $60 / 40$ & $19.95 \pm 13.08$ \\
\hline Mean age \pm SD [years]: & $18.64 \pm 8.56$ & $28.55 \pm 19.23$ & $18.40 \pm 10.72$ \\
\hline Male & $18.76 \pm 6.33$ & $28.65 \pm 19.58$ & $15.05 \pm 19.13$ \\
\hline Mean SALT (AA)/VASI (NSV) \pm SD & $18.44 \pm 11.85$ & $28.41 \pm 19.54$ & $\mathrm{n} / \mathrm{a}$ \\
\hline
\end{tabular}

SD - standard deviation, SALT - Severity of Alopecia Tool, VASI - Vitiligo Area Scoring Index, n/a - not applicable. 
patients with AA, 26 had patchy AA (SALT: S1 $\geq$ S4a) and 7 had severe disease (SALT: $S 4 b \geq S 5)$. All of the 30 enrolled patients with vitiligo had NSV.

IL-17 serum levels were significantly elevated in AA patients and NSV patients compared to healthy controls but were not significantly different between AA and NSV patients (Figure 1). In AA patients IL-17 mean concentration \pm SD was $117.59 \pm 23.83 \mathrm{pg} / \mathrm{ml}$, in NSV $124.82 \pm 27.47 \mathrm{pg} / \mathrm{ml}$. We found no significant sex difference in cytokines levels of female and male patients with AA and NSV.

There was no significant correlation found between serum cytokine levels and extent (SALT/VASI), activity or disease duration, personal history of atopy or autoimmune disorders and family history of AA/vitiligo (Table 2).

\section{Discussion}

Previous studies support the theory of the involvement of IL-17 cells in the pathogenesis of human autoimmune diseases [9]. Production of IL-17 is mainly operated by $T$ helper type 17 (Th17), CD8+ T cells, $\gamma \delta$ T cells, invariant natural killer T cells (iNKT), natural killer (NK) cells, natural Th17 cells, and lymphoid tissue inducer (LTi) cells [10].

IL-17 exerts many physiological functions including neutrophil recruitment, Th2 stimulation to provide an effective response against extracellular organisms, macrophage production of IL-1 $\beta$ and TNF- $\alpha$, and inflammatory mediator matrix metalloproteinases (MMPs) induction. Despite the important role of IL-17 cytokine in regulating adaptive and innate immune systems, its overproduction could be involved in several diseases [4].

\section{Alopecia areata}

In this study, we showed that the levels of Th17- (IL17A)-related cytokines were higher in sera of AA and NSV patients compared to healthy controls. It may imply the role of this population of lymphocytes in both disease development and biology.

Recent data highlighted the role of Th17 cells in the pathogenesis of alopecia areata (AA) Tanemura et al. [11] found the infiltration of CD4(+)IL-17A(+) Th17 cells in the dermis, particularly around hair follicles in AA patients. Han et al. [12] showed that Th17 levels were significantly

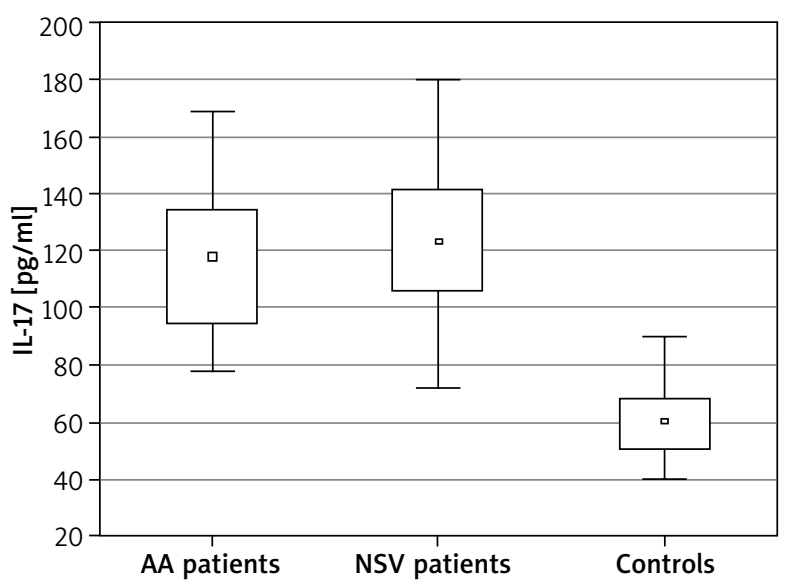

Figure 1. Median serum levels of interleukin (IL)-17 [pg/ml] among patients with alopecia areata (AA), patients with non-segmental vitiligo (NSV) and healthy controls. The Mann-Whitney test revealed a statistically significant difference in serum IL-17 concentrations between AA patients and NSV patients compared to healthy controls

higher in AA patients, whereas Treg levels were lower by comparison. Furthermore, Th17 levels in patients with disease of short duration or the active phase were significantly higher, relative to their respective counterparts. Th17 levels also negatively correlated with disease duration.

Tembhre et al. [13] revealed that the serum cytokine levels of IL-2, IFN- $\gamma$, IL-13, and IL-17A were significantly increased, and serum TGF- $\beta 1$ levels were significantly decreased in patients with AA compared with controls. Those findings suggested altered T-helper cell function, and reduced serum TGF- $\beta 1$ levels suggested a defect in Treg function.

Serum IL-17A in a study by El-Morsy et al. [14] was also significantly higher in AA patients than in control subjects. Serum IL-17A was influenced by the patient age and age of onset of AA but does not seem to influence disease severity.

Elela et al. [15] showed that tissue and serum levels of IL-17, tissue levels of IL-22, and B cell-activating factor (BAFF) were significantly higher in AA patients. Loh et al. [16] identified that lesional IL-17 and IL-22 were signifi-

Table 2. The correlation between serum levels of interleukin (IL)-17 [pg/ml] and extent, activity, and duration of the disease among patients with alopecia areata (AA) and patients with non-segmental vitiligo (NSV)

\begin{tabular}{lcccccc}
\hline $\begin{array}{l}\text { Correlation } \\
\text { between serum } \\
\text { cytokine levels } \\
\text { and }\end{array}$ & $\begin{array}{c}\text { Extent of AA } \\
\text { (SALT) }\end{array}$ & $\begin{array}{c}\text { Activity of AA } \\
\text { (VIDA) }\end{array}$ & Duration of AA & $\begin{array}{c}\text { eExtent of NSV } \\
\text { (VASI) }\end{array}$ & $\begin{array}{c}\text { Activity of NSV } \\
\text { (VIDA) }\end{array}$ & Duration of NSV \\
\hline $\begin{array}{l}\text { IL-17 (q in } \\
\text { Spearman test/ } \\
p \text {-value) }\end{array}$ & $0.248 / 0.163262$ & $0.100 / 0.579343$ & $-0.266 / 0.148211$ & $0.248 / 0.163262$ & $0.003 / 0.989529$ & $-0.153 / 0.428113$ \\
\hline
\end{tabular}

SALT - severity of alopecia tool, VIDA - vitiligo disease activity, VASI - Vitiligo Area Severity Index, IL - interleukin. 
cantly increased in AA patients. Moreover, positive correlations were shown between lesional IL-17, IL-22, and disease severity.

Atwa et al. [17] showed significantly higher levels of serum IL-6, IL-17, IL-21, IL-22, and TNF- $\alpha$. Bain et al. [18] identified increased levels of IL-1 $\beta, I L-6$, and TNF- $\alpha$ in patients with AA. In Tabara et al. [19] study the serum IL-6, IL-15, IL-17A, and IFN- $\gamma$ levels were significantly increased in patients with AA compared with control subjects. The serum cytokine level of IL-17A was found to be decreased when the duration of the current episode was longer than 2 years, but the correlation between the IL-17A serum level and duration of the current episode was not confirmed in the Spearman test.

Guttman-Yassky et al. [20] studied the efficacy and safety of the IL-17A antagonist secukinumab in AA. They conducted a double-blinded, randomized prospective pilot study in which 11 subjects were treated with either secukinumab $(n=7)$ or placebo $(n=4)$. Unfortunately in the secukinumab group, five subjects had no change in response to treatment, one subject had some hair regrowth and one subject had worsening hair loss.

\section{Vitiligo}

Recent research focused on the inflammatory mechanisms involved in vitiligo, suggesting an important role of immune dysregulation in its pathogenesis. Acharya and Mathur [21] have recently conducted a systematic review and meta-analysis of the IL-17 level in patients with vitiligo. A total of 11 case-control studies with 626 vitiligo patients and 475 healthy controls were included. The results confirmed that vitiligo patients have significantly elevated IL-17 levels.

Bhardwaj et al. [22] suggested that increased levels of IL-17A and IL-1 $\beta$ cytokines and decreased expression of MITF may have a possible role in dysregulation of melanocytic activity in the lesional skin and hence might be responsible for the progression of active vitiligo.

Gholijani et al. [23] showed that the levels of all innate immunity (IL-1 $\alpha, \mathrm{IL}-1 \beta, \mathrm{IL}-6, \mathrm{IL}-8, \mathrm{IL}-12, \mathrm{IL}-15$, and TNF- $\alpha$ ), Th1 (IL-2, IFN- $\gamma$, TNF- $\beta$ ) and Th17- (IL-17, IL-23)related cytokines were higher in sera of vitiligo patients than healthy individuals.

A single-arm pilot study using secukinumab in active non-segmental vitiligo was also conducted. The results were disappointing. During the follow-up period, 7/8 patients showed paradoxically signs of further depigmentation while 2 patients showed limited repigmentation. Additional analyses were performed to confirm the immunophenotyping results of the clinical trial pointing to a different role of Th17 cells compared Th17.1 lymphocytes. These experiments point to the delicate Th1/ Th17.1/Th17 balance in non-segmental vitiligo. While IL-17 inhibition did not demonstrate convincing results in active vitiligo, targeted modulation that interacts with the differentiation of Th17 toward Th17/1 and Th1 may be a new and promising approach [24].

\section{Conclusions}

To the best of our knowledge, there is no study comparing the levels of IL-17 in sera of AA and NSV patients. Our results provided evidence on a functional role of Th17 and IL-17 in the pathogenesis of AA and vitiligo and supported the hypothesis that both diseases may share common etiopathogenetic pathways. Imbalance of T cell subpopulations and complex systemic cytokine profiles may contribute to the pathogenesis of AA and vitiligo. These results highlighted the potential therapeutic role by targeting Th17 for the treatment of AA and vitiligo. However, further studies are needed before a definite conclusion can be formulated.

\section{Acknowledgments}

This work was supported by the Medical University of Lodz, Poland (grant numbers 502-03/1-137-04/502-14-39218; 503/1-137-04/503-11-001-18; 503/5-064-01/503-01).

\section{Conflict of interest}

The authors declare no conflict of interest.

\section{References}

1. Rajabi F, Drake LA, Senna MM, et al. Alopecia areata: a review of disease pathogenesis. Br J Dermatol 2018; 179: 103348.

2. Trüeb RM, Dias MFRG. Alopecia areata: a comprehensive review of pathogenesis and management. Clin Rev Allergy Immunol 2018; 54: 68-87.

3. Alikhan A, Felsten LM, Daly M, et al. Vitiligo: a comprehensive overview: Part I. Introduction, epidemiology, quality of life, diagnosis, differential diagnosis, associations, histopathology, etiology, and work-up. J Am Acad Dermatol 2011; 65: 473-91.

4. Bernardini N, Skroza N, Tolino E, et al. IL-17 and its role in inflammatory, autoimmune, and oncological skin diseases: state of art. Int J Dermatol 2020; 59: 406-11.

5. Tomaszewska K, Kozłowska M, Kaszuba A, et al. Increased serum levels of IFN- $\gamma$, IL-1 $\beta$, and IL-6 in patients with alopecia areata and nonsegmental vitiligo. Oxid Med Cell Longev 2020; 2020: 5693572.

6. Olsen EA, Hordinsky MK, Price VH, et al. Alopecia areata investigational assessment guidelines - Part II. J Am Acad Dermatol 2004; 51: 440-7.

7. Hamzavi I, Jain H, McLean D, et al. Parametric modeling of narrow band UV-B phototherapy for vitiligo using a novel quantitative tool: the Vitiligo Area Scoring Index. Arch Dermatol 2004; 140: 677-83.

8. Njoo MD, Das PK, Bos JD, et al. Association of the Kobner phenomenon with disease activity and therapeutic responsiveness in vitiligo vulgaris. Arch Dermatol 1999; 135: 407-13.

9. Dardalhon V, Korn T, Kuchroo VK, et al. Role of Th1 and Th17 cells in organ-specific autoimmunity. J Autoimmun 2008; 31: 252-6. 
10. Amatya N, Garg AV, Gaffen SL. IL-17 signaling: the Yin and the Yang. Trends Immunol 2017; 38: 310-22.

11. Tanemura A, Oiso N, Nakano M, et al. Alopecia areata: infiltration of Th17 cells in the dermis, particularly around hair follicles. Dermatology 2013; 226: 333-6.

12. Han YM, Sheng YY, Xu F, et al. Imbalance of T-helper 17 and regulatory T cells in patients with alopecia areata. J Dermatol 2015; 42: 981-8.

13. Tembhre MK, Sharma VK. T-helper and regulatory T-cell cytokines in the peripheral blood of patients with active alopecia areata. Br J Dermatol 2013; 169: 543-8.

14. El-Morsy EH, Eid AA, Ghoneim H, et al. Serum level of interleukin-17A in patients with alopecia areata and its relationship to age. Int J Dermatol 2016; 55: 869-74.

15. Elela MA, Gawdat HI, Hegazy RA, et al. B cell activating factor and T-helper 17 cells: possible synergistic culprits in the pathogenesis of alopecia areata. Arch Dermatol Res 2016; 308: 115-21.

16. Loh SH, Moon HN, Lew BL, et al. Role of Thelper 17 cells and $T$ regulatory cells in alopecia areata: comparison of lesion and serum cytokine between controls and patients. J Eur Acad Dermatol Venereol 2018; 32: 1028-33.

17. Atwa MA, Youssef N, Bayoumy NM. T-helper 17 cytokines (interleukins 17, 21, 22, and 6, and tumor necrosis factor- $\alpha$ ) in patients with alopecia areata: association with clinical type and severity. Int J Dermatol 2016; 55: 666-72.

18. Bain KA, McDonald E, Moffat F, et al. Alopecia areata is characterized by dysregulation in systemic type 17 and type 2 cytokines, which may contribute to disease-associated psychological morbidity. Br I Dermatol 2020; 182: 130-7.

19. Tabara K, Kozłowska M, Jędrowiak A, et al. Serum concentrations of selected proinflammatory cytokines in children with alopecia areata. Adv Dermatol Allergol 2019; 36: 63-9.

20. Guttman-Yassky E, Nia JK, Hashim PW, et al. Efficacy and safety of secukinumab treatment in adults with extensive alopecia areata. Arch Dermatol Res 2018; 310: 607-14.

21. Acharya P, Mathur M. Interleukin-17 level in patients with vitiligo: a systematic review and meta-analysis. Australas J Dermatol 2020; 61: e208-12.

22. Bhardwaj S, Rani S, Srivastava N, et al. Increased systemic and epidermal levels of IL-17A and IL-1 $\beta$ promotes progression of non-segmental vitiligo. Cytokine 2017; 91: 153-61.

23. Gholijani N, Yazdani MR, Dastgheib L. Predominant role of innate pro-inflammatory cytokines in vitiligo disease. Arch Dermatol Res 2019; 312: 123-31.

24. Speeckaert R, Mylle S, van Geel N. IL-17A is not a treatment target in progressive vitiligo. Pigment Cell Melanoma Res 2019; 32: 842-7. 